\title{
On a neglected variable in theories of pictorial perception: Truncation of the visual field
}

\author{
MARGARET A. HAGEN, REBECCA K. JONES, and EDWARD S. REED \\ Boston University, Boston, Massachusetts 02215
}

\begin{abstract}
Theoretical analyses of pictorial perception have concentrated on the consequences of conflicting flatness and depth information in pictures, but have failed to consider the perceptual effects of the truncation of the visual field attendant on any pictorial display. The importance of this variable both for methods of testing pictorial information and for theory building was demonstrated. Under four different viewing conditions, adults were asked to scale the size and distance of five isoceles triangles at five different distances. The four conditions were unobstructed static monocular view, peephole view, view through a rectangular frame, and view of all the stimuli photographed in a slide. The slopes of the peephole, truncation, and slide conditions' scaling functions were all significantly smaller than the slope of the untruncated Monocular condition, and the Y-intercepts were all greater. A decrease in overconstancy in the size functions indicated a similar effect for all three truncated conditions. Results are interpreted as supporting the hypothesis that truncation of the visual field, both in pictures and in peephole views of the real world, causes a frontal shift in the localization of the visible field, with a resultant compression of perceived size and distance.
\end{abstract}

It has often been observed, particularly in the crosscultural literature, that pictorial perception of the third dimension is inferior to the perception of depth in the ordinary environment (Deregowski, 1972; Gibson, 1969; Wilcox \& Teghtsoonian, 1971; Yonas \& Hagen, 1973). This finding is frequently attributed to the coexistence in pictures of competing flatness and depth information (Attneave \& Frost, 1969; Gibson 1969; Gregory, 1970; Hochberg, 1962; Schlosberg, 1941; Yonas \& Hagen, 1973). Surface texture, binocular and monocular parallax, and other cues all specify the picture surface as flat and unextended in depth, while the monocular distance cues of size, linear and texture perspective all specify a surface layout extended in depth.

However, the attention paid to this very critical coexistence of flatness and depth information overlooks what may be another very important characteristic of pictures in terms of determining depth perception. This characteristic is the truncation of the visual field, particularly of the foreground, necessarily entailed by the limited size of ordinary pictorial images. Hagen and Teghtsoonian (Note 1) determined that the monocular distance scaling function

This research was supported by a grant from the National Institute of Mental Health to the first author, Grant 1-RO1MH27947-01. The authors gratefully acknowledge the conceptual assistance of Martha Teghtsoonian and Abigail Stewart in the formulation of these studies. The authors also wish to thank José Valdes for his assistance in the conduct of this study. Requests for reprints should be sent to Margaret A. Hagen, 64 Cummington Street, Department of Psychology, Boston University, Boston, Massachusetts 02215. for real scenes across distances of 6 to 50 in. (15.2 to $127 \mathrm{~cm}$ ) was linear, with a slope of 0.626 and a y-intercept of zero. (With head motion the slope is 0.56 , without, 0.69.) Since a constancy function for absolute value judgments would have a slope of $1.0, \mathrm{y}=\mathrm{x}$, this mean monocular function represents a compression of perceived distance relative to physical distance with the anchor point at 0 . In the present experiment, the monocular visual field was truncated in two ways, and the effects on the resulting scaling function were determined. In one condition, the field was truncated by use of a peephole (which is often used to control head motion); in the other condition, the field was truncated with a rectangular occluding frame. The size and shape of the frame were determined by life-size projections of slides taken of the field. These two truncated real scene conditions were compared both to a slide condition and to an untruncated real scene condition. In addition to distance judgments, size judgments were also obained in order to test the effects of truncation on the scaling of size. It was hypothesized that the obtained functions in the truncated conditions would reflect the compressed distance perception so common to pictures taken with lenses longer than $50 \mathrm{~mm}$. This increasing distance compression is readily observable across a series of pictures taken with increasingly longer lenses (see, e.g., pp. 6-7 in Time-Life Camera Buyer's Guide). Thus, the phenomenon is well documented but poorly explained. The present experiment hypothesized that this distance compression was due to the lack of available optical information for foreground dis- 
tance. In the ordinary environment, the visual field begins at the face. If, in pictorial or truncated perception, the same assumption is made, then the relatively slow rate of change of visual angle with distance must necessarily specify relatively short distances. The purpose of the present study was to test for the operation of the ordinary visual field boundary assumption on the perception of distance under conditions of truncated view and to compare the obtained functions to those generated in both truncated real scene and slide conditions. Specifically, a slope flatter than .69 was predicted as reflective of "telephoto" distance compression in the expected linear function. In addition, increased variability of the intercept was also predicted, since there was no information available in the truncated display to consistently fix an anchor point across subjects.

\section{METHOD}

\section{Subjects}

Sixty volunteers from introductory psychology classes participated in the experiment. There were 15 subjects assigned randomly to each of the four conditions. About half of the subjects in each condition were male and about half were female.

\section{Apparatus and Procedure}

Stimuli to be judged for distance and size were presented vertically on a $4 \times 8 \mathrm{ft}(1.22 \times 2.44 \mathrm{~m})$ flat textured table top. The stimuli were isoceles triangles whose height and base were $2,3,4,5$, and 6 in. $(5.08,7.62,10.16,12.7$, and $15.24 \mathrm{~cm})$. They were cut from Day-Glo red-orange posterboard and mounted upright with L-shaped hardware. The table top was covered with black, white, and gray checked gingham stretched tight over $3 / 4-i n$. $(1.91-\mathrm{cm})$ plywood. The entire table was surrounded by 4 -ft $(1.22-\mathrm{m})$ high off-white curtains suspended from the ceiling which occluded the view of everything in the room but the stimulus field. Perceived distance always referred to the distance between a small ( $2 \mathrm{~cm}$ in diameter) circular marker placed flat on the table $18 \mathrm{in} .(45.75 \mathrm{~cm})$ from the subject and the bases of the triangles presented vertically $6,10.2,17.3,29.5$, and $50 \mathrm{in}$. $(15.24,25.9,44,75,127 \mathrm{~cm})$ beyond the marker. At each of the five distances, the subjects made five distance judgments to the bases of the five different sized triangles as well as a judgment of the height of each of the five triangles at each of the five distances. Thus, every subject was given 25 stimulus presentations at which $s /$ he judged both the size and the distance of the presented triangles. Half the time, size was judged first, and half the time, distance was judged first. This order was randomized as was the order of the 25 presentations for each subject. This design produced five judgments from each subject for every size and every distance.

\section{Viewing Conditions}

All four viewing conditions were monocular, with each subject indicating his/her preferred eye. The nonpreferred eye was covered with an eyepatch. In the untruncated monocular condition, the eye was positioned $6 \mathrm{in}$. $(15.24 \mathrm{~cm})$ above the table and 18 in. $(45.75 \mathrm{~cm})$ from the circular marker. Head motion was constrained by use of a chinrest with temple guides, Biometrika Model 115-4. The subjects viewed the stimulus field through a $35 \times 15 \mathrm{~cm}$ slot, which allowed proper orientation without truncating the field of view. In the peephole condition, a $4 \times 4 \mathrm{ft}(1.2 \times 1.2 \mathrm{~m})$ Masonite sheet covered the viewing end of the table with a viewing aperture, $2 \mathrm{~mm}$ in diameter, placed at the station point de- scribed above. In the rectangular truncation condition, view was constrained by a small slot $(6.5 \times 4.5 \mathrm{~cm})$ placed $5 \mathrm{~cm}$ in from the station point. The size and shape of the available visual field was identicai to that in the fourth condition, slides. These slides were taken in color with a 50-mm SLR camera placed at the station point described above. The dimensions of the truncated field were determined by projecting the slides until the images were identical to those obtained when viewing the real scenes. In both the rectangular truncation and the slide conditions, view was constrained not only by the cardboard frame, but by the headrest as well.

The subjects were given the following instructions: "This is an experiment in visual perception. We wish to study peoples' judgments of size and distance. We will ask you to open the curtain and look closely through the slot (peephole). In front of you is a (picture of a) table. Each time on the table there will be an orange circle and a triangle. When

(the experimenter) asks you to make a distance judgment, you may open the curtain and look around once through the slot (peephole) for as long as you please, and close the curtain. Then, using this tape measure, you should try to duplicate the distance between the center of the circle and the base of the triangle. We wish you to hold the measure in your right hand, pulling the tape out with your left hand (like this ) so that the numbers are not showing. If you were to lay the tape on the table after you make your judgment, one end would be at the circle, the other at the base of the triangle. For size we want you to do essentially the same thing. That is, judge so that if you placed the measure at the base of the triangle with the tape straight up it would duplicate the height to the apex of the triangle. Please hold the tape measure right in front of you so that you can see it, then turn it so that the numbers may be read by the experimenter. To return the tape just push the button."

\section{RESULTS}

In general, the hypothesis that perceived distance would be compressed by the operation of visual field truncation received good support. The slopes of all of the truncated distance functions were significantly lower than the slope of the untruncated monocular control condition. In a like manner, the overestimation of size observed in the control condition was diminished by truncation. The hypothesis of increased variability of intercept in the truncated conditions was not supported.

\section{Distance Judgments}

In order to calculate mean functions for each condition, the means of each of the five distance judgments were determined for each subject and used as data to compute group functions, which are graphed in Figure 1. Since observation of the group functions indicated the appropriateness of linear functions as descriptive of the data, the least squares technique was used to fit a straight line function to the mean distances judged for every subject, yielding slope, y-intercept, and coefficient of determination. The mean slopes intercepts and coefficients of determination for each condition are tabulated in Table 1 . As can be seen from Table 1, the very high $r^{2} s$ are indicative of the goodness of fit of the linear functions. These group linear functions are graphed in Figure 2. 


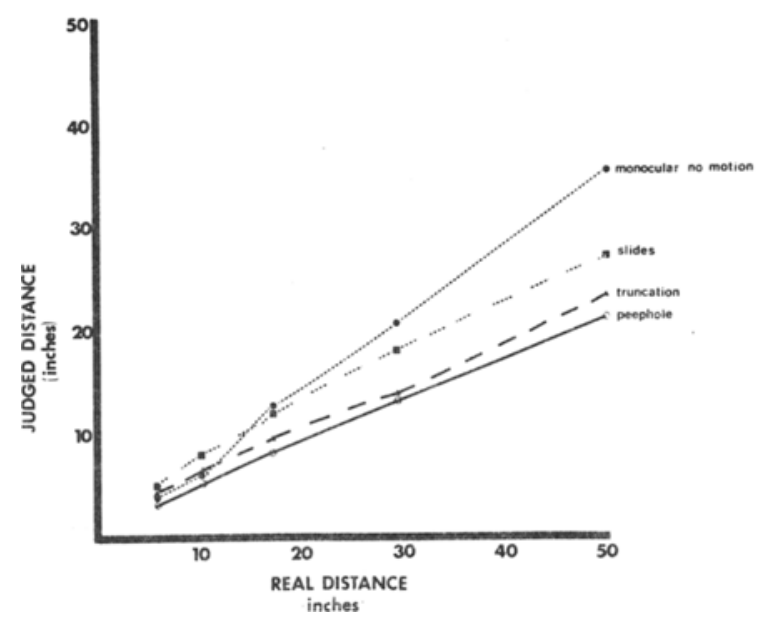

Figure 1. Group functions plotted on mean distances judged.

The obtained scores were then analyzed in three one-way analyses of variance with condition as the independent variable and slope, intercept, and $\mathrm{r}^{2}$ as the dependent variables. In the analysis of the slope data, the main effect for condition was significant, $F(3,56)=3.99, p<.05$. Duncan post hoc comparisons showed that the untruncated monocular slope was significantly greater than the other three which did not differ, $\mathrm{p}<.05$. These slope values were tested individually against the hypothetical value of 1.0, and all four were found to be significantly smaller, with at least $\mathrm{p}<.001$. In the analysis of the intercept data, the main effect for condition was also significant, $F(3,56)=4.31, p<.01$. Duncan post hoc comparisons indicated that the intercept for the slide condition was significantly greater than those for the monocular and peephole conditions, $p<.05$. The truncated condition intercept lay between, but was not significantly different from, the others. Individual $t$ tests showed that all the intercepts except that of the monocular function were significantly greater than 0 , at least at the $\mathrm{p}<.02$. Analysis of variance of $r^{2}$ showed no significant mean effect.

\section{Size Judgments}

Again, group means and linear functions with coefficients of determination were calculated for

Table 1

Linear Functions for Distance with Attendant Coefficients of Determination

\begin{tabular}{lll}
\hline Condition & Function & $\mathrm{r}^{2}$ \\
\hline Monocular & $\mathrm{y}=.69+.60$ & .99 \\
Truncated & $\mathrm{y}=.44+2.02$ & .99 \\
Peephole & $\mathrm{y}=.41+1.33$ & .99 \\
Slide & $\mathrm{y}=.49+3.42$ & .98 \\
\hline
\end{tabular}

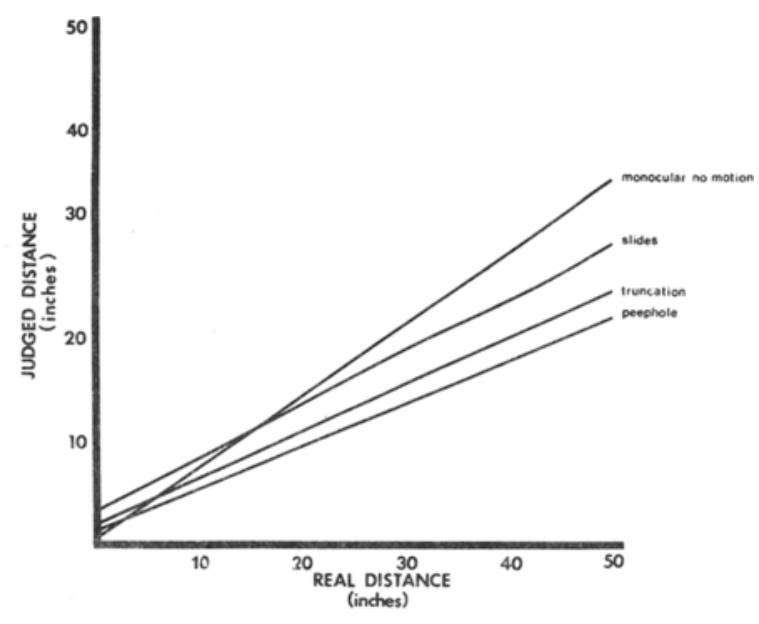

Figure 2. Straight-line functions of mean fitted distance functions.

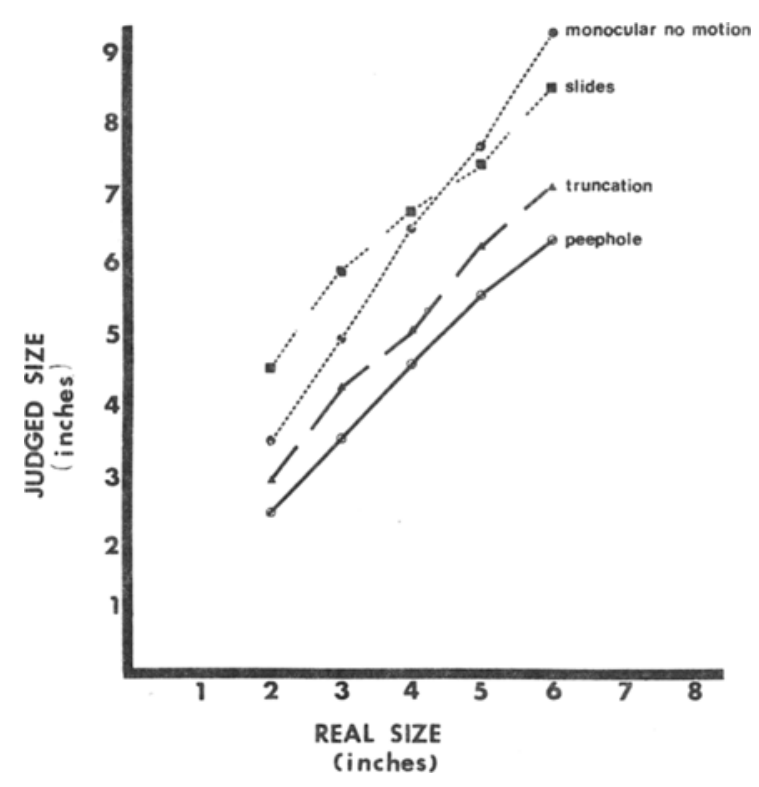

Figure 3. Group functions plotted on mean sizes judged,

each condition. The mean sizes are graphed in Figure 3, and the linear functions appear in Figure 4. The linear functions with $r^{2} s$ are tabulated in Table 2 .

Again, individual linear functions fitted to each subject's data yielded, by regression, both slope and intercept. Coefficients of determination were also calculated. Three one-way analyses of variance were performed. The analysis of the slope data yielded no significant main effect for condition, $F(3,56)=1.06$, $\mathrm{p}>.05$. Individual $t$ tests against the hypothetical slope value of 1.0 showed that only the monocular slope was significantly greater that $1.0, \mathrm{p}<.05$. The analysis of the intercept data yielded a significant 
main effect for condition, $F(3,56)=9.36, p<.01$. Duncan post hoc comparisons indicated that the intercept for the slide condition was significantly greater than the other three intercepts, which did not differ from each other, $\mathrm{p}<.01$. Individual $t$ tests against the hypothetical value of 0.0 showed that both the slide intercept and the peephole intercepts were significantly greater than 0.0 , with at least $\mathrm{p}<.05$. The analysis of variance for $\mathrm{r}^{2}$ also showed a significant main effect for condition, $F(3,56)=3.27$, $\mathrm{p}<.05$. Duncan post hoc comparisons showed that $\mathrm{r}^{2}$ for the slide condition, .89, was significantly smaller than $\mathrm{r}^{2}$ for the other three conditions, .96, .95 , and .95 . Observation of the intercept data for both size and distance failed to yield any evidence of increased variability of intercepts as a function of condition. (See Table 3.)

It was hypothesized that as a function of truncation of the foreground of the visual field, distance perception would be compressed in a telephoto manner yielding scaling functions with slopes significantly flatter than those obtained with untruncated monocular static view. This hypothesis was supported. The slopes of the peephole, truncated, and slide conditions were all significantly smaller than the slope of the monocular condition, indicating a telephoto-like compression of apparent distance. It is interesting to note that observation of this distortion of apparent distance has ordinarily been confined to photographs taken with lenses longer than the ordinary $50 \mathrm{~mm}$. The present investigation, however, supports the argument that all photographs and peepholes will produce a similar distance distortion to the extent that the foreground from scene to observer is truncated by image size, frame, or viewing aperture. The diminished overconstancy in the size scaling for the truncation similarly supports this hypothesis of compressed perceived distance. It had

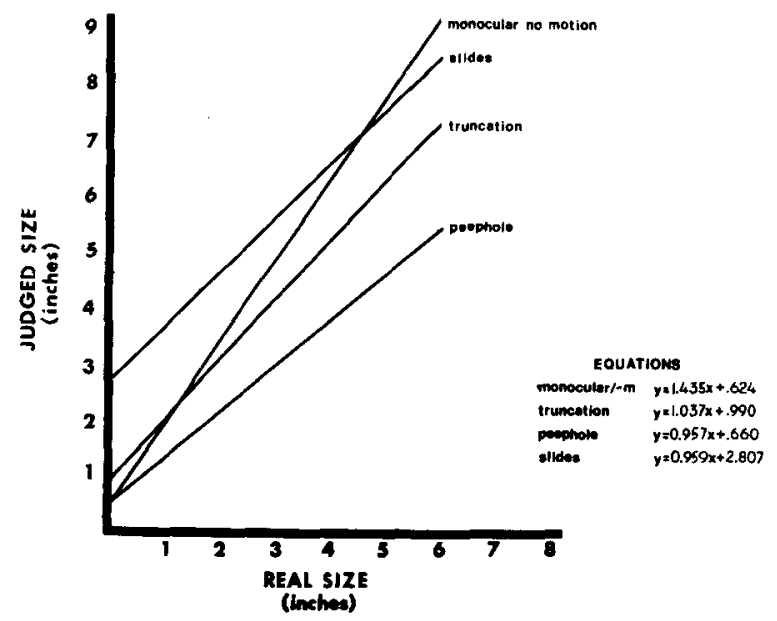

Figure 4. Straight-line functions of mean fitted size functions.
Table 2

Linear Functions for Size with Attendant Coefficients of Determination

\begin{tabular}{lll}
\hline Condition & \multicolumn{1}{c}{ Function } & $\mathrm{r}^{2}$ \\
\hline Monocular & $\mathrm{y}=1.44+.62$ & .96 \\
Truncated & $\mathrm{y}=1.04+.99$ & .95 \\
Peephole & $\mathrm{y}=.96+.66$ & .94 \\
Slide & $\mathrm{y}=.96+2.81$ & .89 \\
\hline
\end{tabular}

Table 3

Intercepts for Size and Distance by Condition

\begin{tabular}{lccccc} 
& \multicolumn{2}{c}{ Size } & & \multicolumn{2}{c}{ Distance } \\
\cline { 5 - 6 } \cline { 5 - 6 } Condition & Mean & SD & & Mean & SD \\
\hline Monocular & .62 & .97 & .60 & 2.11 \\
Truncated & .99 & .88 & 2.02 & 2.05 \\
Peephole & .66 & .80 & 1.33 & 1.79 \\
Slide & 2.81 & 2.02 & 3.50 & 2.83 \\
\hline
\end{tabular}

also been hypothesized that in the absence of visual information specifying the amount of truncated foreground the intercepts of the distance functions would be extremely variable. Observation of Table 3 provides no support for this hypothesis. What occurred instead was the generation of intercepts in the truncated conditions all significantly greater than 0 . Moreover, the intercept for the slide condition tended to be still higher than the intercepts of the real scene truncated conditions. It appears as if, in the absence of specific information for foreground distance, observers simply apply some small fixed value to all of their distance judgments. Moreover, they do this with considerable intersubject consistency, such that the slide distance intercept of 3.42 is significantly greater than both the monocular, .60, and the peephole, 1.33 , intercept. The only explanation for such consistency is the observers' knowledge that the field consisted of an ordinary table in an ordinary room (or picture of one). Thus, the scales of the five judgments are in accord with such an assumption. It would be interesting to see where observers anchor the scale in the face of complete projective ambiguity.

\section{REFERENCE NOTE}

1. Hagen, M. A., \& Teghtsoonian, M. Are formally equivalent sources of information perceptually equivalent? Paper presented at the Eastern Psychological Association Annual Meeting, Boston, April 1977.

\section{REFERENCES}

Attneave, F., \& Frost, R. The determination of perceived tridimensional orientation by minimum criteria. Perception \& Psychophysics, 1969, 6, 391-396.

Deregowski, J. B. Pictorial perception and culture. Scientific American, 1972, 227, 82-88.

Gibson, E. J. Principles of perceptual learning and development. New York: Appleton-Century-Crofts, 1969. 
GrEGoRY, R. L. The intelligent eye. New York: McGraw-Hill, 1970.

Hochberg, J. E. Psychophysics of pictorial perception. AudioVisual Communication Review, 1962, 10, 22-54.

Schlosberg, H. Stereoscopic depth from single pictures. American Journal of Psychology, 1941, 54, $601-605$.

Wilcox, R. L., \& Teghtsoonian, M. The control of relative size by pictorial depth cues in children and adults. Journal of Experimental Child Psychology, 1971, 11, 413-429.
Yonas, A., \& Hagen, M. A. Effects of static and kinetic depth information on the perception of size in children and adults. Journal of Experimental Child Psychology, 1973, 15, 254-265.

(Received for publication June 2, 1977; revision accepted November 25,1977 .) 